\title{
Usando Comunicação Aumentativa e Alternativa para ensinar Equivalência de Estímulos
}

\author{
Jayr Pereira ${ }^{1}$, Natália Franco ${ }^{1}$, Rosany Pereira ${ }^{2}$, Robson Fidalgo $^{1}$ \\ ${ }^{1}$ Centro de Informática - Universidade Federal de Pernambuco (UFPE) \\ Recife - PE - Brazil \\ \{jap2, nmf, rdnf\}@cin.ufpe.br \\ ${ }^{2}$ Centro Educacional Nasília Ferreira Dantas \\ Penaforte - CE - Brazil \\ rosanya30@gmail.com
}

\begin{abstract}
In the context of inclusive education, Augmentative and Alternative Communication Systems (AAC) can be used for various purposes. In this sense, this article proposes a conceptual framework for adaptating activities based on the theory of Equivalence of Stimuli for AAC systems. This proposal was evaluated by health and education professionals, based on the technology acceptance model (TAM), obtaining positive results, thus showing its great potential for acceptance.
\end{abstract}

Resumo. No contexto de educação inclusiva, sistemas de Comunicação Aumentativa e Alternativa (CAA) podem ser usados para diversos fins. Neste sentido, este artigo propõe um framework conceitual para adaptação de atividades baseadas na teoria de Equivalência de Estímulos para sistemas de CAA. Essa proposta foi avaliada por profissionais das áreas de saúde e educação, com base no modelo de aceitação da tecnologia (TAM), obtendo resultados positivos, mostrando assim seu grande potencial de aceitação.

\section{Introdução}

A Comunicação Aumentativa e Alternativa (CAA) [ASHA 2019] é uma Tecnologia Assistiva importante nas adaptações necessárias para pessoas com deficiência intelectual e/ou dificuldades na fala (e.g., pessoas com Paralisia Cerebral, Microcefalia, Transtornos do Espectro Autista, sequelas de AVC e Apraxia). Essas pessoas, apresentam limitações na comunicação gestual, oral e/ou escrita e não conseguem ter uma comunicação funcional. Para esses casos, a CAA, a partir da seleção de imagens com legendas, ajuda a: 1) desenvolver a compreensão; 2) reduzir a frustração na tentativa de se comunicar; 3) ter um poder maior de escolha; e 4) expressar sentimentos e opiniões. Além da comunicação, a CAA também pode ser usada como uma ferramenta de suporte ao desenvolvimento de atividades pedagógicas para a educação inclusiva desses indivíduos. No contexto da educação inclusiva, a CAA pode ser usada para diversos fins, por exemplo: 1) Adaptação de conteúdo - ensino de letras, sílabas, números e operações aritméticas, bem como outros conceitos (e.g., animais, cores e lugares); 2) Contação de histórias e reprodução de músicas - ensino de novos conceitos a partir de histórias ou músicas; 3) Instrução de rotinas - ensino de rotinas (e.g., "escovar os dentes" e "tomar banho"); 4) Equivalência de Estímulos - ensino de novos conceitos e de relações entre conceitos. 
A capacidade de formar classes de estímulos equivalentes a partir de relações arbitrárias entre estímulos é fundamental para o desenvolvimento da comunicação funcional e da leitura com compreensão [de Rose 1993]. Neste contexto, o paradigma de Equivalência de Estímulos [Sidman and Tailby 1982], a partir de procedimentos de escolha de acordo com o modelo (do inglês, Matching to Sample), tem sido utilizado para ensinar discriminações condicionais que permitem o estabelecimento de relações arbitrárias entre estímulos diferentes (e.g., comer $\rightarrow$ comida; comida $\rightarrow$ bolo). Incluindo a emergência de novas relações a partir das relações condicionais ensinadas anteriormente (e.g., comer $\rightarrow$ bolo). Assim, o paradigma fornece uma fundamentação teórica e sistemática para a definição empírica do que é "compreensão" [de Rose and Bortoloti 2007], o que permite que ele seja usado não só como ferramenta de ensino, mas também como meio de avaliação.

Em Equivalência de Estímulos, o procedimento de escolha de acordo com o modelo consiste em um conjunto de atividades nas quais o indivíduo é exposto a estímulos de modelo e de comparação, que são apresentados em forma de figuras, texto ou sons, elementos que estão geralmente presentes em sistemas de CAA. Nesses sistemas, pictogramas (i.e., figuras) são utilizados como suporte visual para a construção de frases. Cada pictograma está atrelado a uma palavra, que por sua vez pode ser visualizada pelo usuário e falada pelo sintetizador de voz, comumente presente nesses sistemas. No entanto, o uso desses sistemas na realização de atividades de Equivalência de Estímulos requer um alto nível de personalização, possibilitando que o mediador (i.e., terapeuta, professor, cuidador ou pai/mãe) crie atividades temáticas (e.g., números, letras, animais, rotinas). A plataforma aBoard [Franco et al. 2017], é um exemplo de sistema de CAA que possibilita a personalização de conteúdo, dando ao mediador a liberdade de adicionar e excluir pictogramas, criar e excluir pastas, editar os rótulos de pictogramas e pastas, etc.

Sendo assim, explorando a capacidade de personalização de sistemas como o $a B o-$ ard, este trabalho propõe um framework conceitual para a adaptação de atividades de Equivalência de Estímulos em sistemas de CAA. Esse framework conta com seis tipos de atividades que podem ser adaptadas não só para o aBoard, mas para qualquer ferramenta de CAA que possibilite a personalização de conteúdo e que conte com duas áreas explicitamente distintas, uma para mostrar os estímulos modelo e outra para mostrar os estímulos de comparação. Para fins de prova de conceito, essas atividades foram ilustradas em um sistema de CAA fictício e sua aceitação avaliada junto a profissionais de saúde e educação, usando como base o modelo de aceitação de tecnologia (TAM). Os resultados obtidos mostram que a proposta é bem aceita pelos profissionais, considerando como métrica o grau de concordância com as proposições presentes no instrumento de pesquisa.

O restante deste artigo está organizado da seguinte maneira: na Seção 2 apresentase uma visão geral da plataforma $a$ Board, da teoria de Equivalência de Estímulos e do modelo de aceitação de tecnologia (TAM); na Seção 3 é apresentada a proposta de adaptação de atividades de equivalência de estímulos em CAA; na Seção 4 apresenta-se o método utilizado na avaliação; na Seção 5 são mostrados os resultado; e, finalmente, na Seção 6 as considerações finais. 
VIII Congresso Brasileiro de Informática na Educação (CBIE 2019)

Anais do XXX Simpósio Brasileiro de Informática na Educação (SBIE 2019)

\section{Fundamentação Teórica}

\subsection{Plataforma aBoard}

A Figura 1 mostra uma visão geral da Plataforma aBoard [Franco et al. 2017], que é uma plataforma em nuvem para CAA e educação inclusiva. A plataforma é composta por três componentes: 1) aplicativo aBoard $^{1}$, usado por pessoas com dificuldades na fala para comunicação por meio de um dispositivo móvel; 2) aBoard editor, usado por mediadores (i.e., terapeuta, professor, cuidador ou pai/mãe) para customizar o vocabulário que será usado no aplicativo; e 3) aBoard server, que é usado para o armazenamento dos dados, controle de acesso e disponibilização do conteúdo. Seu diferencial é a tecnologia baseada em computação em nuvem e a característica de sugerir símbolos e oferecer respostas rápidas. O primeiro permite ao usuário acessar seus dados de qualquer dispositivo, e o segundo permite a sugestão de um novo pictograma de acordo com os pictogramas previamente selecionados e o fácil uso de expressões básicas (e.g., sim, não, obrigado) sem interferir na construção de uma expressão. Além disso, o aBoard fornece uma plataforma que: 1) está disponível offline, não exigindo uma conexão com a Internet para usar o dispositivo móvel; 2) é baseada em uma interface minimalista, que mantém o usuário focado no que é importante: os pictogramas; 3) organiza o conteúdo em categorias, o que torna mais rápida a seleção de pictogramas, reduzindo o tempo de busca; 4) está na nuvem, permitindo acesso aos dados do usuário de qualquer dispositivo; 5) fornece feedback visual e sonoro ao selecionar qualquer pictograma ou botão de ação, agindo como um reforço para o usuário; 6) permite a criação de expressões explícitas (e.g., frases e operações matemáticas) em uma área de frase; e 7) permite a customização do conteúdo, o que aumenta a identificação entre usuário e dispositivo, incentivando sua adoção.

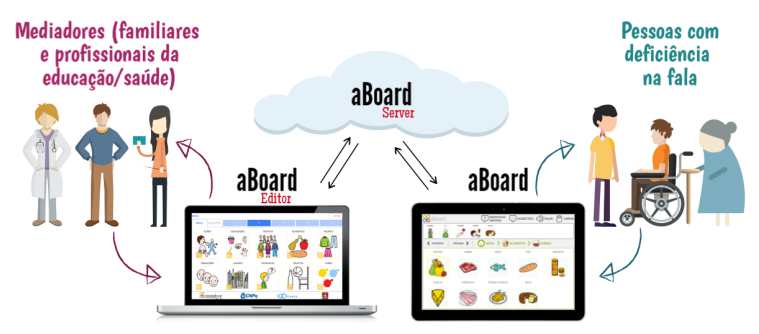

Figura 1. Visão geral da plataforma aBoard.

Observe que, uma vez que a personalização de conteúdo é feita fora do dispositivo móvel e na nuvem, a Plataforma aBoard permite que o mediador realize essa atividade em qualquer computador com acesso à Internet e sem retirar o dispositivo móvel do usuário. Além disso, mesmo que o computador ou o dispositivo móvel seja danificado, o conteúdo salvo na nuvem sempre poderá ser acessado de qualquer outro computador ou dispositivo móvel. Isso dá ao mediador a liberdade de personalizar o conteúdo do vocabulário de acordo com as situações do dia-a-dia do usuário, conforme o avanço de suas capacidades de comunicação e aquisição de vocabulário.

\subsection{Equivalência de Estímulos}

Pensamento e linguagem exigem a capacidade de agrupar estímulos em classes, que emergem de relações entre estímulos que nunca foram explicitamente ensinados. Ou seja, a

\footnotetext{
${ }^{1}$ Disponível na Google Play Store
} 
VIII Congresso Brasileiro de Informática na Educação (CBIE 2019)

Anais do XXX Simpósio Brasileiro de Informática na Educação (SBIE 2019)

Equivalência de Estímulos [Sidman and Tailby 1982] consiste em estabelecer um tipo de relação de significado entre os símbolos (i.e., imagens, conceitos, letras, sílabas, palavras ou sons) e os eventos ou fenômenos aos quais esses símbolos se referem. Um dos procedimentos mais utilizados para estabelecer relações entre estímulos é o Matching to Sample (MTS) [Sidman 1994]. Nesse procedimento, um estímulo modelo (SM) é apresentado junto com alguns estímulos de comparação. Dentre os estímulos de comparação, tem-se apenas um considerado correto, chamado de estímulo discriminativo (SD), enquanto que os demais (distratores), são chamados de estímulos delta $(\mathrm{S} \Delta$ ). Desta forma, uma relação condicional é estabelecida entre dois estímulos, sendo um de modelo e outro de comparação. Por exemplo, se A1 é mostrado, então escolha B1; se A2 é mostrado, então escolha B2, e assim por diante. Uma variação da correspondência típica é a correspondência multi-modelo, onde a quantidade de modelos de estímulos é maior do que um, o que permite mais de uma correspondência ao mesmo tempo.

Segundo [Sidman 1994], o pareamento de estímulos pode dar origem a relações de equivalência, isto é, a partir do ensino explícito de relações entre estímulos $(\mathrm{A} 1 \rightarrow \mathrm{B} 1$, $\mathrm{B} 1 \rightarrow \mathrm{C} 1)$, outras relações podem emergir sem a necessidade de instrução direta $(\mathrm{A} 1 \rightarrow \mathrm{C} 1)$ [Sidman 1994]. Note que as classes de estímulos equivalentes são baseadas nas relações de equivalência provenientes da teoria dos conjuntos: reflexividade (A1 $\leftrightarrow$ A1) simetria $(\mathrm{A} 1 \rightarrow \mathrm{B} 1, \mathrm{~B} 1 \rightarrow \mathrm{C} 1)$, e transitividade $(\mathrm{A} 1 \rightarrow \mathrm{C} 1)$ [Sidman and Tailby 1982]. A Figura 2, ilustra a aplicação dessas relações em um conjunto de atividades de equivalência de estímulos que seguem o modelo Matching to Sample. Na figura, a relação de reflexividade acontece quando o indivíduo é exposto a um estímulo modelo e um estímulo discriminativo, que devem ser exatamente iguais (e.g., comer $\leftrightarrow$ comer; comida $\leftrightarrow$ comida; e bolo $\leftrightarrow$ bolo). Já a relação de simetria se dá quando o sujeito é exposto a um estímulo modelo e a pelo menos um de comparação que contenham características em comum, ou seja, sejam da mesma classe (e.g., comer $\rightarrow$ comida; comida $\rightarrow$ bolo). A transitividade consiste na relação que deve emergir sem a necessidade de treinamento explícito (e.g., comer $\rightarrow$ bolo).

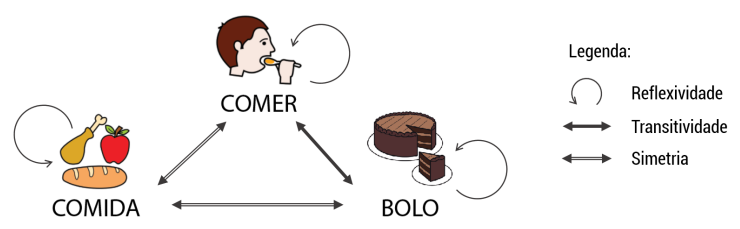

Figura 2. Exemplo de atividade de equivalência de estímulos.

\subsection{TAM (Technology Acceptance Model)}

O TAM (Technology Acceptance Model) [Davis 1985] provê explicações de fatores que são considerados determinantes na aceitação de tecnologia [Davis et al. 1989]. Esse modelo é utilizado como ferramenta de avaliação na academia e na indústria, tendo como objeto de investigação tecnologias de diversos tipos (e.g., softwares, dispositivos e plataformas). O TAM possibilita a investigação de impressões a respeito da aceitação de tecnologia de usuários de diversas populações, como profissionais da saúde [Chau and $\mathrm{Hu} 2002$ ] e educação [Teo 2009], por exemplo.

O modelo postula que a aceitação de uma determinada tecnologia é explicada por quatro fatores básicos [Davis et al. 1989], que são definidos por [Holden and Karsh 2010] 
VIII Congresso Brasileiro de Informática na Educação (CBIE 2019)

Anais do XXX Simpósio Brasileiro de Informática na Educação (SBIE 2019)

da seguinte maneira: Atitude (ATT), é o julgamento avaliativo de um indivíduo sobre o comportamento alvo em alguma dimensão (e.g., bom/ruim, prejudicial/benéfico); Intenção Comportamental de Uso (BI), a motivação ou disposição de um indivíduo em se esforçar para realizar o comportamento desejado; Utilidade Percebida (PU), é a percepção de um indivíduo de que o uso de determinada tecnologia aumentará o desempenho do trabalho; e Facilidade de Uso Percebida (PEOU), é a percepção de um indivíduo de que o uso de determinada tecnologia será livre de esforço.

\section{Adaptação de Atividades de Equivalência de Estímulos}

Com base no paradigma de Equivalência de Estímulos, é proposto um framework conceitual que conta com seis tipos de atividades que podem ser adaptadas em sistemas de CAA. As principais características dessas atividades são: 1) apresentar um grau de dificuldade crescente; 2) explorar as relações de reflexividade, simetria e transitividade; e 3) empregar correspondência multi-modelo para ordem de trabalho e sequência entre estímulos discriminativos.

O framework proposto pode ser aplicado em qualquer sistema de CAA, desde que este tenha uma área de conteúdo e uma área para criação de frases. Conforme exemplo da Figura 3, a área de conteúdo (delimitada por um retângulo grande) será usada para mostrar os estímulos de comparação (discriminativo e delta), enquanto que a área de frase (delimitada por um retângulo pequeno) será usada para mostrar os estímulos modelos e as respostas. Este artigo apresenta o aBoard como uma alternativa que atende esses critérios, mas o uso dessa proposta não se limita a esse sistema. A Figura 3-A mostra um modelo de atividade sem o estímulo delta. A Figura 3-B ilustra uma atividade com o estímulo delta. Por fim, a Figura 3-C apresenta um modelo de atividade com multi-modelo.

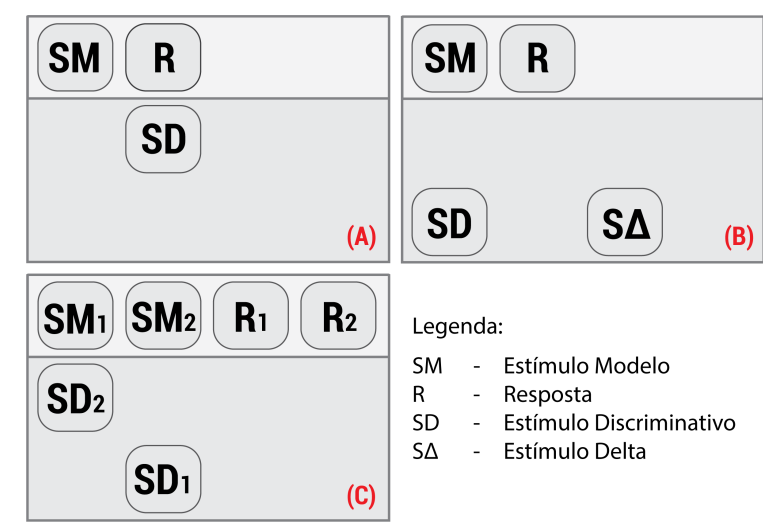

Figura 3. Modelos de atividades adaptadas.

As primeiras cinco atividades baseiam-se na correspondência típica (i.e., apenas um modelo de estímulo é apresentado) e exploram as relações de reflexividade, simetria ou transitividade da discriminação condicional com ou sem estímulo delta. Por sua vez, a última atividade é baseada na correspondência multi-modelo (i.e., mais de um modelo de estímulo) que, além de permitir a exploração das relações de reflexividade, simetria e transitividade da discriminação condicional, permite também trabalhar com ordem/sequência entre estímulos discriminativos. As seis atividades, em ordem crescente de dificuldade, são apresentadas a seguir: 
A1 - Descriminação reflexiva sem estímulo delta: nesta atividade, o sujeito é exposto a um único estímulo modelo e a um único estímulo de comparação. Ambos os estímulos devem ser iguais para mostrar para o sujeito que se trata da mesma coisa.

A2 - Discriminação reflexiva com estímulo delta: diferente da atividade A1, nesta atividade o sujeito é exposto a mais de um estímulo, onde apenas um deles é o discriminativo, sendo o outro o estímulo delta. O objetivo desta atividade é avaliar se a relação de reflexividade foi adquirida pelo sujeito.

A3 - Discriminação simétrica: assim como na atividade A1, o sujeito é exposto a um único estímulo modelo e a um único estímulo de comparação. A diferença está no objetivo que é mostrar ao sujeito que diferentes estímulos podem ser equivalentes. Para isso, o estímulo discriminativo precisa ter características comuns ao estímulo modelo.

A4 - Discriminação simétrica com estímulo delta: as diferenças entre esta atividade e a atividade A3 são: 1) quantidade de estímulos de comparação, que devem ser pelo menos dois, onde apenas um deles será o estímulo discriminativo e 2) o objetivo, que é avaliar se a relação de simetria foi adquirida pelo sujeito.

A5 - Transitividade discriminativa com estímulo delta: a diferença entre esta atividade e a atividade A4 está em seu objetivo, pois esta visa avaliar se um novo par de estímulos, a partir de um estímulo comum, emergiu sem a execução de uma nova atividade.

A6 - Discriminação multi-modelo: as diferenças entre esta atividade e todas as anteriores são: 1) quantidade de estímulos modelos, que devem ser pelo menos dois e 2) o objetivo, que é ensinar e avaliar se a ordem/sequência entre os estímulos foi adquirida pelo sujeito. Note que os estímulos delta são inerentes a esta atividade, uma vez que, mesmo sendo a quantidade de estímulos modelo e de comparação iguais, qualquer erro na sequência de discriminação produz um estímulo delta.

Destaca-se que, antes do início da atividade, o mediador deve ter a atenção do sujeito, pois ele deve observar que o mediador selecionará estímulo modelo na área de conteúdo e então este aparecerá na área de frase. Na sequência, o mediador deve estimular o sujeito a selecionar o estímulo discriminativo que está localizado na área de conteúdo. Se isso levar mais de quinze segundos, o mediador deve dar uma dica verbal ou física e esperar mais quinze segundos. Se ocorrer novamente, o mediador deve avaliar o assunto e decidir se termina a seção, altera a atividade ou dá um tempo de descanso ao sujeito. Ressalta-se que um protocolo para aplicar essas atividades está além do escopo deste trabalho. Destaca-se ainda que, a posição dos estímulos comparativos deve mudar em todas as atividades, que podem ser usadas para explorar a relação entre vários conceitos, por exemplo: 1) imagem para palavra escrita; 2) imagem para palavra ditada; 3) palavra escrita para palavra ditada; 4) imagem ou palavra para sua primeira letra; 5) imagem ou palavra para sua primeira sílaba; 6) imagem ou palavra para algumas (ou todas) suas letras (considerando ou não a sequência correta); 7) imagem ou palavra para algumas (ou todas) as suas sílabas (considerando ou não a sequência correta); 8) número por quantidade; 9) sequência de etapas em uma rotina; e 10) sequência de elementos de uma música. 
VIII Congresso Brasileiro de Informática na Educação (CBIE 2019)

Anais do XXX Simpósio Brasileiro de Informática na Educação (SBIE 2019)

\section{Avaliação da Aceitação}

\subsection{Amostragem}

A amostra desta pesquisa se caracteriza como uma amostra por conveniência e não constitui uma amostra probabilística, pois não havia critérios estatísticos para a seleção de participantes. Assim, foram selecionados profissionais das áreas de saúde e educação que trabalham com pessoas com deficiência intelectual e têm experiência no uso de CAA e/ou equivalência de estímulos. Ao todo, foram selecionados 23 profissionais, distribuídos em 5 áreas de atuação, sendo: 7 psicólogos, 6 fonoaudiólogos, 6 educadores, 3 psicopedagogos e 1 assistente terapêutico.

\subsection{Instrumento e procedimentos}

O instrumento de pesquisa utilizado é dividido em duas partes: (i) um questionário demográfico com 7 (sete) perguntas para identificação do participante; e (ii) um questionário com 15 (quinze) questões assertivas (cf. Tabela 1) com uma escala Likert de 5 (cinco) pontos, sendo: 1) Discordo Totalmente $(D T), 2)$ Discordo Parcialmente $(D P), 3)$ Nem discordo, nem concordo $(N), 4)$ Concordo Parcialmente $(C P)$; e 5) Concordo Totalmente $(C T)$. As questões assertivas são baseadas no questionário proposto por [Chau and Hu 2002] e contemplam as dimensões listadas e definições apresentadas por [Holden and Karsh 2010] para cada fator do TAM.

Tabela 1. Questões assertivas do instrumento

\begin{tabular}{|c|c|}
\hline Código & Assertiva \\
\hline ATT1 & Eu acho que realizar atividades de equivalência de estímulos com CAA é uma boa ideia. \\
\hline ATT2 & Eu acho que realizar atividades de equivalência de estímulos com CAA é benéfico. \\
\hline ATT3 & $\begin{array}{l}\text { Eu acho que realizar atividades de equivalência de estímulos com CAA tornaria as sessões } \\
\text { terapêuticas mais interessantes. }\end{array}$ \\
\hline ATT4 & Eu gostaria de realizar atividades de equivalência de estímulos com CAA. \\
\hline BI1 & Eu pretendo realizar atividades de equivalência de estímulos com CAA regularmente. \\
\hline $\mathrm{BI} 2$ & Eu sou favorável a realizar atividades de equivalência de estímulos com CAA. \\
\hline $\mathrm{BI} 3$ & Eu realizaria atividades de equivalência de estímulos com CAA. \\
\hline PU1 & $\begin{array}{l}\text { Eu acho que realizar atividades de equivalência de estímulos com CAA não vai melhorar } \\
\text { meu trabalho durante a terapia. }\end{array}$ \\
\hline PU2 & $\begin{array}{l}\text { Eu acho que realizar atividades de equivalência de estímulos com CAA vai aumentar a } \\
\text { eficácia da terapia. }\end{array}$ \\
\hline PU3 & $\begin{array}{l}\text { Eu acho que realizar atividades de equivalência de estímulos com CAA vai facilitar meu } \\
\text { trabalho durante a terapia. }\end{array}$ \\
\hline PU4 & $\begin{array}{l}\text { Eu acho que realizar atividades de equivalência de estímulos com CAA não é útil para o } \\
\text { processo terapêutico. }\end{array}$ \\
\hline PEOU1 & $\begin{array}{l}\text { Eu acho que realizar atividades de equivalência de estímulos com CAA não é viável para o } \\
\text { processo terapêutico. }\end{array}$ \\
\hline PEOU2 & Eu teria dificuldade em aprender a realizar atividades de equivalência de estímulos com CAA. \\
\hline PEOU3 & Eu teria dificuldade para realizar atividades de equivalência de estímulos com CAA. \\
\hline PEOU4 & $\begin{array}{l}\text { Eu teria dificuldade em tornar-me habilidoso na realização de atividades de equivalência de } \\
\text { estímulos com CAA. }\end{array}$ \\
\hline
\end{tabular}

Antes que os participantes respondessem os questionários, lhes foi apresentado o conjunto de atividades descrito na Seção 3 ilustrado em um sistema de CAA fictício. Além disso, todos os participantes foram informados sobre os objetivos da pesquisa, e avisados que ao responder o questionário estariam de acordo com o termo de consentimento da 
VIII Congresso Brasileiro de Informática na Educação (CBIE 2019)

Anais do XXX Simpósio Brasileiro de Informática na Educação (SBIE 2019)

pesquisa, que garante a anonimidade dos seus dados e os deixa livres para escolher se querem ou não participar ou ter seus dados computados.

\subsection{Método de análise}

Para a análise dos resultados das escalas utilizadas nesta pesquisa, foi utilizado o Grau de Concordância de cada Preposição $(G C P)$, que é baseado no oscilador estocástico de Wilder Jr., também conhecido como indicador de força relativa [Wilder 1978]. Para isso, se fez necessário calcular o número de participantes que concordam $($ Con $)-C o n=$ $C T+C P+\frac{N}{2}-$ e discordam $(D i s)-D i s=D T+D P+\frac{N}{2}-$ de cada proposição para, assim, chegar ao grau de concordância $-G C P=100-\left(\frac{100}{\frac{C o n}{D i s}+1}\right)$. Outra métrica utilizada é o Grau de Concordância do Fator (GCF), que consiste na média dos GCP das questões referentes a cada fator. De maneira que a média dos GCP das questões BI1, BI2 e BI3, por exemplo, equivale ao GCF do fator Intenção Comportamental de Uso. Os valores de GCP e GCF obtidos são interpretados conforme tabela proposta por [Davis 1976] (cf. Tabela 2).

Tabela 2. Interpretação de valores do GCP

\begin{tabular}{|l|l|}
\hline Valor do Grau de Concordância & Interpretação adequada \\
\hline 90 ou mais & Concordância muito forte \\
\hline 80 a 89,99 & Concordância substancial \\
\hline 70 a 79,99 & Concordância moderada \\
\hline 60 a 69,99 & Concordância baixa \\
\hline 50 a 59,99 & Concordância desprezível \\
\hline 40 a 49,99 & Discordância desprezível \\
\hline 30 a 39,99 & Discordância baixa \\
\hline 20 a 29,99 & Discordância moderada \\
\hline 10 a 19,99 & Discordância substancial \\
\hline 9,99 ou menos & Discordância muito forte \\
\hline
\end{tabular}

\section{Resultados}

A Tabela 3 apresenta os resultados da aplicação do método de análise descrito na Seção 4.3, com a quantidade de concordantes (Con) e de discordantes (Dis), assim como o grau de concordância da proposição (GCP) e o grau de concordância do fator (GCF). As questões marcadas com asterisco (*) são de escala inversa. O GCP das questões inversas foram calculados subtraindo 100 do resultado final da equação do GCP.

Como mostrado na Tabela 3, todos os fatores tiveram grau de concordância muito forte, considerando os níveis propostos na Tabela 2. O fator Atitude (ATT) obteve GCF de 98,91 , que significa que a avaliação dos participantes sobre o comportamento alvo (i.e., usar a proposta) é positivo. O alto valor obtido para fator Intenção Comportamental de Uso (BI) $(93,48)$, sugere que os participantes estão motivados e dispostos a usar a proposta. Quanto ao fator de Utilidade Percebida (PU), o alto valor obtido $(95,65)$ demonstra que os participantes têm a percepção de que o uso da proposta vai afetar positivamente o seu trabalho, tornando-o mais fácil e eficaz. O fator de Facilidade de Uso Percebida (PEOU) também obteve um GCF alto $(95,11)$, demonstrando a percepção dos participantes de que o uso da proposta não demanda um alto nível de esforço. 
VIII Congresso Brasileiro de Informática na Educação (CBIE 2019)

Anais do XXX Simpósio Brasileiro de Informática na Educação (SBIE 2019)

Tabela 3. Resultados

\begin{tabular}{|c|c|c|c|c|c|c|c|c|c|}
\hline Questão & DT & DP & $\mathbf{N}$ & CP & CT & Con & Dis & GCP & GCF \\
\hline ATT1 & 0 & 0 & 0 & 3 & 20 & 23 & 0 & 100 & \multirow{4}{*}{98,91} \\
\hline ATT2 & 0 & 0 & 0 & 4 & 19 & 23 & 0 & 100 & \\
\hline ATT3 & 0 & 0 & 2 & 7 & 14 & 22 & 1 & 95,65 & \\
\hline ATT4 & 0 & 0 & 0 & 6 & 17 & 23 & 0 & 100 & \\
\hline BI1 & 0 & 0 & 5 & 12 & 6 & 20,5 & 2,5 & 89,13 & \multirow{3}{*}{93,48} \\
\hline $\mathrm{BI} 2$ & 0 & 0 & 1 & 4 & 18 & 22,5 & 0,5 & 97,83 & \\
\hline $\mathrm{BI} 3$ & 0 & 1 & 1 & 2 & 19 & 21,5 & 1,5 & 93,48 & \\
\hline PU1* & 20 & 3 & 0 & 0 & 0 & 0 & 23 & 100 & \multirow{4}{*}{95,65} \\
\hline PU2 & 0 & 0 & 3 & 10 & 10 & 21,5 & 1,5 & 93,48 & \\
\hline PU3 & 0 & 0 & 5 & 9 & 9 & 20,5 & 2,5 & 89,13 & \\
\hline PU4* & 20 & 3 & 0 & 0 & 0 & 0 & 23 & 100 & \\
\hline PEOU1* & 22 & 1 & 0 & 0 & 0 & 0 & 23 & 100 & \multirow{4}{*}{95,11} \\
\hline PEOU2* & 18 & 2 & 3 & 0 & 0 & 1,5 & 21,5 & 93,48 & \\
\hline PEOU3* & 18 & 2 & 3 & 0 & 0 & 1,5 & 21,5 & 93,48 & \\
\hline PEOU4* & 18 & 2 & 3 & 0 & 0 & 1,5 & 21,5 & 93,48 & \\
\hline
\end{tabular}

As proposições que obtiveram menor GCP foram BI1 e PU3 (89,13 ambas), isso se dá pela quantidade de respostas neutras $(\mathrm{N})$. A proposição BI1, por exemplo, está relacionada ao fator de Intenção Comportamental de Uso e está interessada em saber a intenção do participante em usar a proposta regularmente. Como a proposta foi apresentada por meio de um sistema de CAA fictício, os participantes podem ter percepções limitadas a respeito do uso real, e assim, preferem não opinar sobre. PU1, por sua vez, está relacionada ao fator de Utilidade Percebida e está interessada na percepção do indivíduo sobre a capacidade da proposta em melhorar o seu trabalho, e também pode ter sido afetada pelo uso de um sistema fictício.

\section{Considerações finais}

Este artigo propõe um framework conceitual que conta com seis tipos de atividades de Equivalência de Estímulos que podem ser adaptadas em um sistema de Comunicação Aumentativa e Alternativa (CAA). Essas atividades exploram os conceitos de reflexividade, simetria, transitividade e descriminação multi-modelo, com base no modelo Matching to Sample. A proposta de adaptação foi ilustrada em um sistema de CAA fictício e apresentada a profissionais das áreas de saúde e educação, com o objetivo de avaliar a aceitação da proposta, usando como base o modelo de aceitação de tecnologia (TAM).

Os resultados obtidos indicam que a proposta é bem aceita pelos profissionais, que são seus eventuais usuários, considerando os fatores propostos pelo TAM: Atitude, Intenção Comportamental de Uso, Utilidade Percebida e Facilidade de Uso Percebida. Essa conclusão baseia-se no grau de concordância obtido por cada proposição do questionário aplicado, e no grau de concordância de cada fator estudado.

O framework aqui proposto pode ser usado para a adaptação de atividades que podem ser utilizadas em diversos contextos educacionais. Os benefícios que podem ser obtidos a partir de sua exploração dependem do grau de personalização permitido pelo sistema de CAA utilizado e da criatividade do mediador. De forma geral, o uso do framework proposto favorece: 1) o aumento do número de possíveis conceitos a serem ensinados; e 2) a exploração dos elementos de interação do CAA (i.e., figuras, texto e sons), 
VIII Congresso Brasileiro de Informática na Educação (CBIE 2019)

Anais do XXX Simpósio Brasileiro de Informática na Educação (SBIE 2019)

aumentando assim o as possibilidades durante as atividades.

Como trabalhos futuros, pretende-se explorar os elementos de jogos presentes nas atividades que compõem o framework e inserir outros elementos que sejam necessários para caminhar em direção de um jogo sério para o ensino de equivalência de estímulos usando recursos de CAA.

\section{Agredecimentos}

Esta pesquisa é apoiada pelo CNPq (458798/2013-4 e 461492/2014-8) e pela CAPES (88882.347547/2019-01).

\section{Referências}

ASHA (2019). American speech-language-hearing association.

Chau, P. Y. and Hu, P. J.-H. (2002). Investigating healthcare professionals' decisions to accept telemedicine technology: an empirical test of competing theories. Information \& management, 39(4):297-311.

Davis, F. D. (1985). A technology acceptance model for empirically testing new enduser information systems: Theory and results. $\mathrm{PhD}$ thesis, Massachusetts Institute of Technology.

Davis, F. D., Bagozzi, R. P., and Warshaw, P. R. (1989). User acceptance of computer technology: a comparison of two theoretical models. Management science, 35(8):9821003.

Davis, J. A. (1976). Levantamento de dados em sociologia. Zahar.

de Rose, J. C. (1993). Classes de estímulos: implicações para uma análise comportamental da cognição. Psicologia: teoria e pesquisa, 9(2):283-303.

de Rose, J. C. and Bortoloti, R. (2007). A equivalência de estímulos como modelo do significado. Acta Comportamentalia: Revista Latina de Análisis de Comportamiento, 15(3):83-102.

Franco, N., Lima, T., Lima, A., Silva, E., Lima, R., Cavalcante, T., and Fidalgo, R. (2017). aboard: Uma plataforma para educação inclusiva a partir de comunicação aumentativa e/ou alternativa. In Brazilian Symposium on Computers in Education (Simpósio Brasileiro de Informática na Educação-SBIE), volume 28, page 977.

Holden, R. J. and Karsh, B.-T. (2010). The technology acceptance model: its past and its future in health care. Journal of biomedical informatics, 43(1):159-172.

Sidman, M. (1994). Equivalence relations and behavior: A research story. Authors Cooperative.

Sidman, M. and Tailby, W. (1982). Conditional discrimination vs. matching to sample: An expansion of the testing paradigm. Journal of the Experimental Analysis of behavior, 37(1):5-22.

Teo, T. (2009). Modelling technology acceptance in education: A study of pre-service teachers. Computers \& Education, 52(2):302-312.

Wilder, J. W. (1978). New concepts in technical trading systems. Trend Research. 